\title{
Particle-in-cell simulations of hollow cathode enhanced capacitively coupled radio frequency discharges
}

T. Lafleur and R. W. Boswell

Citation: Phys. Plasmas 19, 023508 (2012); doi: 10.1063/1.3685709

View online: http://dx.doi.org/10.1063/1.3685709

View Table of Contents: http://pop.aip.org/resource/1/PHPAEN/v19/i2

Published by the American Institute of Physics.

\section{Related Articles}

Radio frequency discharge with control of plasma potential distribution

Rev. Sci. Instrum. 83, 02A720 (2012)

Optimization of gas utilization efficiency for short-pulsed electron cyclotron resonance ion source

Rev. Sci. Instrum. 83, 02A342 (2012)

Hollow target magnetron-sputter-type solid material ion source

Rev. Sci. Instrum. 83, 02B715 (2012)

Numerical study of ion energy and angular distributions in dualfrequency capacitively coupled CF4 plasmas Phys. Plasmas 19, 023506 (2012)

Plasma resonances in a microwave-driven microdischarge

Appl. Phys. Lett. 100, 064102 (2012)

\section{Additional information on Phys. Plasmas}

Journal Homepage: http://pop.aip.org/

Journal Information: http://pop.aip.org/about/about_the_journal

Top downloads: http://pop.aip.org/features/most_downloaded

Information for Authors: http://pop.aip.org/authors

\section{ADVERTISEMENT}

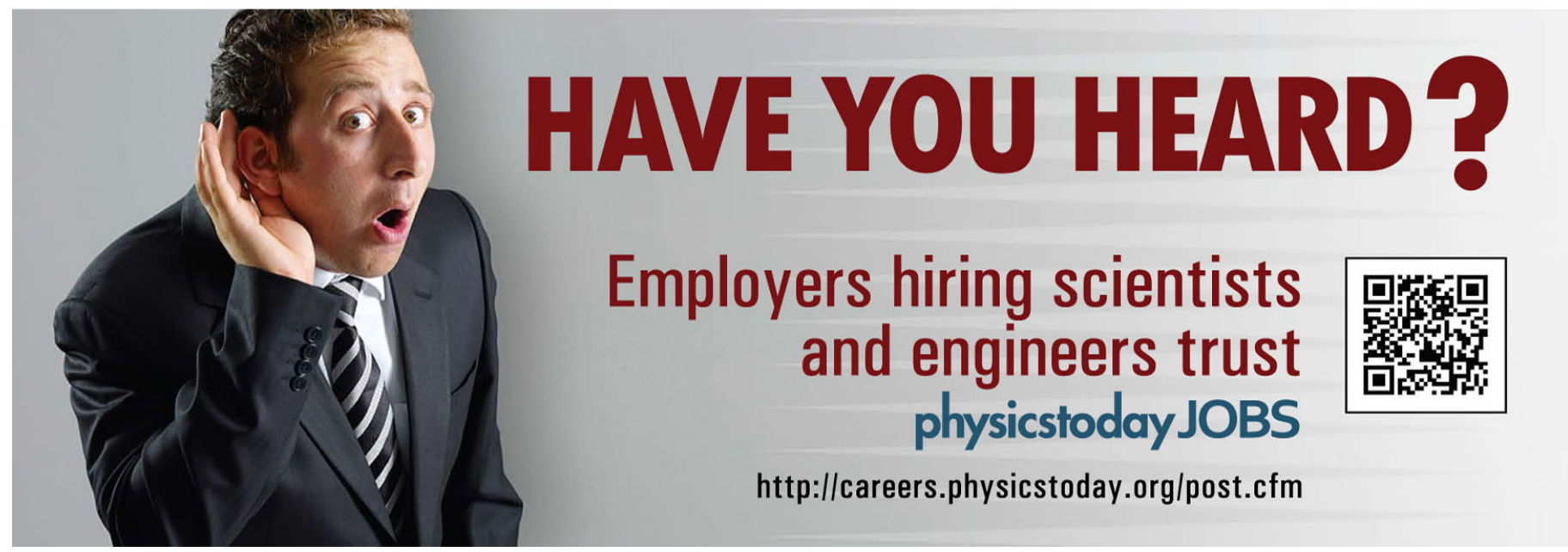




\title{
Particle-in-cell simulations of hollow cathode enhanced capacitively coupled radio frequency discharges
}

\author{
T. Lafleur ${ }^{\mathrm{a})}$ and R. W. Boswell \\ Space Plasma, Power and Propulsion Group, Research School of Physics and Engineering, The Australian \\ National University, Canberra ACT 0200, Australia
}

(Received 3 October 2011; accepted 16 January 2012; published online 22 February 2012)

A two-dimensional particle-in-cell simulation has been developed to study density enhancement of capacitively coupled rf discharges with multi-slit electrodes. The observed density increase is shown to result from a hollow cathode effect that takes place within the multi-slit electrode configuration, which forms as a result of secondary electron emission due to ion bombardment. By investigating the ionization and power deposition profiles, it is found that $\mathrm{rf}$ sheath heating is too weak to sustain the discharge, and that secondary electron acceleration within the sheath is the primary heating mechanism. Due to a capacitive voltage divider formed by the rf sheaths at each electrode, the area ratio of the powered and ground electrodes is observed to have a strong effect on the resulting discharge, and if the ground electrode area is too small, the voltage drop at the powered electrode is too low to sustain a hollow cathode discharge. (C) 2012 American Institute of Physics. [doi:10.1063/1.3685709]

\section{INTRODUCTION}

Hollow cathodes (HCs) are a type of electrode geometry in both dc and rf plasma systems, which allow for the formation of a hollow cathode effect (HCE). ${ }^{1} \mathrm{HC}$ systems typically consist of a small hollow cylindrical or rectangular electrode electrically connected through a power generator to a larger grounded electrode. ${ }^{1,2}$ Depending on the operating conditions (i.e., gas pressure and applied voltage), a HCE can be initiated, which is characterized by a large increase in current density (and hence plasma density) for a given voltage. This large increase in plasma density has consequently made $\mathrm{HCs}$ attractive for a number of applications, including light sources for spectrochemistry, ${ }^{3}$ as ionization and neutralization sources in plasma thrusters, ${ }^{4}$ ion lasers, ${ }^{5}$ and especially as etching and deposition devices in plasma processing. ${ }^{1,2,6-11}$ Here, the $\mathrm{HC}$ often also serves as the input gas nozzle, ${ }^{1}$ and the large plasma densities obtained allow for an increased yield in radical and chemical species formation through gas dissociation. This has been exploited for dry etching of silicon, ${ }^{7}$ thin film processing, ${ }^{2,8}$ deposition of diamond crystals and polycrystalline films, ${ }^{6}$ and treatment of material surfaces. ${ }^{9}$

A number of different physical mechanisms are thought to be important in the operation of both dc and rf HCs. Positive ions bombarding the HC surface produce secondary electrons that subsequently get accelerated across the sheath potential to very high energies. ${ }^{1}$ When these high energy electrons encounter the opposite $\mathrm{HC}$ wall, they are reflected by the repelling potential and, thus, can undergo an oscillatory back-and-forth motion (also called pendulur motion) between the HC walls. ${ }^{12,13}$ These electrons are very efficient ionizers, and because they are electrostatically confined, they can undergo multiple inelastic collisions before being lost to the electrodes. Other mechanisms that are thought to

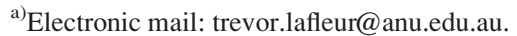

contribute to the $\mathrm{HCE}$ include photoemission of electrons from the $\mathrm{HC}$ by high energy photons produced within the plasma $^{1}$ and thermionic emission of electrons from the $\mathrm{HC}$ walls, ${ }^{4}$ resulting either from heating due to direct ion bombardment or due to the presence of an external heater system. These thermionic HCs are used primarily in plasma thrusters as both an electron source, an ion beam exhaust neutralizer, ${ }^{4}$ and more recently as a separate micro-propulsion system. ${ }^{14,15}$

Because of the small diameter or gap between opposite walls of the HC hole or channel (typically only a few millimeters), experimental information on the electron and ion dynamics within the discharge is limited, or often cannot be directly obtained. While fluid simulations have been used to provide insight into the physical phenomena in these discharges, ${ }^{16-18}$ these types of simulations often make use of simplified electron and ion distribution functions. By contrast, particle-in-cell (PIC) simulations ${ }^{19}$ require no assumptions about the electron and ion distribution functions and, with a reasonable system model, represent fully selfconsistent methods for simulating plasma discharges. Recent PIC simulations of dc HCs have been performed, ${ }^{20,21} \mathrm{dem}-$ onstrating the effect of the pendular electrons ${ }^{20,22}$ and charged particle dynamics, and in particular showing the presence of a number of distinct electron populations within the distribution function. ${ }^{20}$ By contrast, there appears to be no detailed recorded simulations of rf $\mathrm{HC}$ operation, and while these systems are thought to operate in a similar manner to dc HCs, this has not been thoroughly investigated. In particular, it is not known if secondary electron acceleration is the primary heating mechanism or whether rf sheath heating plays an important role, since electrons within the $\mathrm{HC}$ are almost completely surrounded by an oscillating rf sheath.

In this work, we perform two-dimensional self-consistent PIC simulations of a multi-slit capacitively coupled $\mathrm{rf}$ system and demonstrate that the formation of an rf hollow cathode discharge is only possible in the presence of secondary electron emission due to ion bombardment. The resulting 
discharge is characterized, and the ionization and power deposition profiles are studied in detail with the aim of clarifying the electron heating mechanism.

\section{DESCRIPTION OF SIMULATION MODEL}

We use a custom developed two-dimensional explicit PIC simulation code, PHOENIX $2 D$, written in MATLAB using a highly vectorized code structure. Time comparison tests of a one-dimensional version of the code with a similar code written in $\mathrm{C}++$ showed similar run times. Electrons and ions are moved using a leap-frog scheme, ${ }^{19}$ and Poisson's equation is solved on a uniform rectangular grid within the simulation domain during each time step. A standard Monto Carlo null-collision algorithm ${ }^{23}$ is used to simulate neutral collisions with charged particles, and the background neutral gas used is argon, which is assumed to have a constant density and a Maxwellian velocity distribution with a temperature of $0.026 \mathrm{eV}$. Electron-neutral collisions considered include elastic, excitation, and ionization reactions, using standard cross-section data from Refs. 24-27, while ionneutral collisions include elastic and charge-exchange collisions, using cross-section data from Ref. 28.

A simple energy independent model is used to account for secondary electron emission, with a constant emission coefficient of between 0.05 and $0.2 .^{29}$ When an ion or electron leaves the simulation domain across an electrode surface, it is removed from the discharge and a Monto Carlo method is used to determine whether a secondary electron is produced. Secondary electrons are chosen from a halfMaxwellian velocity distribution (with a temperature of $2 \mathrm{eV}$, Ref. 4) and are emitted away from the particular portion of the electrode where they were born.

As the basis for our simulation model, we use a similar electrode configuration to the experimental system in Ref. 10, a schematic of which is shown in Fig. 1(a). In Ref. 10, a density enhancement has been reported in a capacitively coupled plasma (CCP) when using a multi-hole powered electrode instead of a simple plane electrode. This enhancement is affected by the voltage drop at the powered electrode, and also by the electrode material used, ${ }^{30}$ and is thought to occur due to a HCE that is produced within the holes of the electrode. We choose to model a similar type of system here, because the effect of multi-hole/multi-slit and simple plane electrodes can easily be accounted for within the simulation code. To simplify the simulation and reduce run times, we ignore the larger ground chamber and simulate only a small portion of the electrode region, as indicated in Fig. 1(a). The simulation uses a two-dimensional Cartesian coordinate system and, thus, simulates an infinite slab geometry. Due to the presence of multiple slits, we treat the top and bottom simulation boundaries as symmetry planes. The $y$-component of the electric field is zero along these boundaries and particles crossing the open sections of these boundaries are specularly reflected, representing particles entering the simulation domain from an adjacent region. Depending on the system configuration, plane or multi-slit electrodes can be modelled. When simulating a multi-slit electrode, the slit half-width is $2.5 \mathrm{~mm}$, with a depth of $10 \mathrm{~mm}$. The
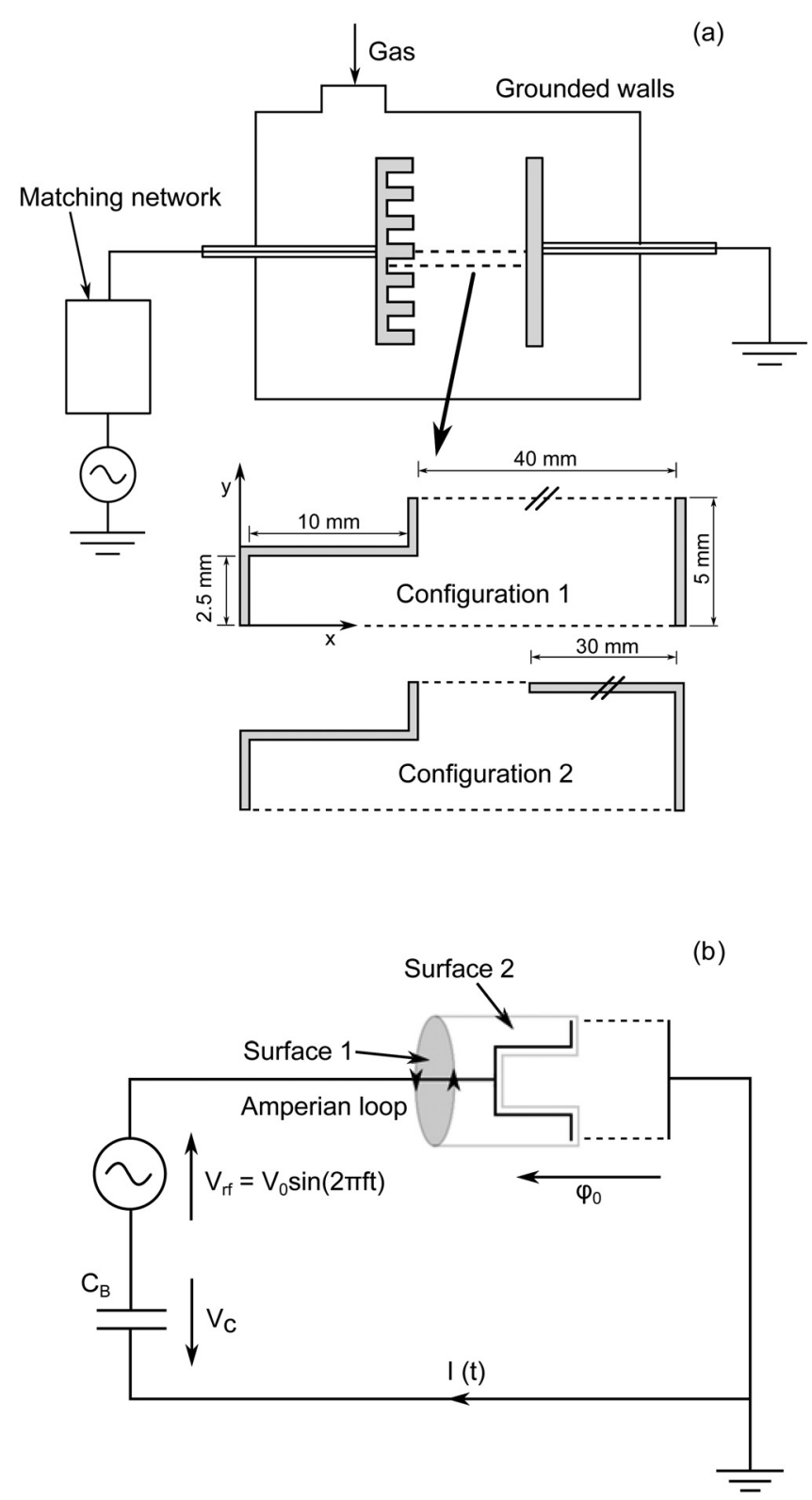

FIG. 1. (a) Schematic of the PIC model, indicating the simulation domain and electrode configurations used. A third electrode configuration (not shown) is also modelled, where the hollow cathode piece in configuration 2 is replaced with a simple plane electrode. (b) External circuit connected to the simulation domain, consisting of an rf voltage source $\left(V_{r f}\right)$ and a bias capacitor $\left(C_{B}\right)$. The total voltage drop across the $\mathrm{HC}$ system is $\phi_{0}$, while the voltage drop across the bias capacitor is $V_{c}$. The Amperian loop and surfaces 1-2 shown are used in calculating the displacement current (see Sec. IV F).

right-hand electrode is grounded and is spaced $40 \mathrm{~mm}$ away from the powered left-hand electrode. From a computational point of view, such a large gap length, together with the high expected densities $\left(>1 \times 10^{17} \mathrm{~m}^{-3}\right.$ when a HCE is present $)$ result in the need for a large number of grid points and a large number of particles to maintain good statistical representation (especially of the high energy secondary electron population). A much smaller gap length (relative to the slit dimensions) such as used in the PIC simulations in Ref. 20 would be more ideal, but as will be discussed later in Sec. IV A, this causes a simulation problem when modelling rf systems. 
Simulations use between about $3 \times 10^{5}-1.2 \times 10^{6}$ ionelectron pairs (i.e., about $6 \times 10^{5}-2.4 \times 10^{6}$ total particles), and each particle represents around $1 \times 10^{7}$ real particles. The time step used is small enough to resolve the electron plasma frequency and is of the order of $1 \times 10^{-11} \mathrm{sec}$; while many tens of thousands of grid points are used in the simulation domain, sufficient to resolve the sheath width and electron Debye length within the sheath. In some of the simulations, the Debye length within the high-density regions at the center of the discharge is not completely resolved, but since the plasma is quasi-neutral in this region, and since radial density gradients are small here, this is not expected to have a large effect. In the simulations, electrons and ions are initially loaded with a uniform distribution, with temperatures of $T_{e}=2 \mathrm{eV}$ and $T_{i}=0.03 \mathrm{eV}$, respectively.

The simulation domain is coupled to an external circuit, as shown in Fig. 1(b). Here, the powered electrode is connected to an rf voltage source which generates a voltage $V_{r f}(t)=V_{0} \sin (2 \pi f t)$ and a bias capacitor $C_{B}$. The frequency of the voltage source is $f=13.56 \mathrm{MHz}$. The bias capacitor is needed to prevent any dc current from flowing between the electrodes and allows the formation of a dc self bias on the powered electrode. The capacitance of the bias capacitor is chosen to be $500 \mathrm{pF}$, which is sufficiently large that only a small fraction of the rf voltage is dropped across it, while small enough so that the charging time is not too long. Because of the presence of the bias capacitor, the voltage on the powered electrode is not known. The circuit and simulation potential are solved using the method in Ref. 31. Here the field solver is decomposed into a solution of the Poisson equation (with zero boundary conditions), and the Laplace equation (with the external circuit and other boundary conditions). Although discussed in detail in Ref. 31, in short, the voltage across the bias capacitor, $V_{c}$, in Fig. 3 is found from Kirchhoff's voltage law (noting the sign convention used in Fig. 1(b)) as

$$
V_{c}=V_{r f}-\phi_{0}
$$

where $\phi_{0}$ is the voltage on the powered electrode. At the ground electrode, from charge conservation, the total charge density, $\sigma_{T}$, on the electrode can be found from

$$
A \frac{d \sigma_{T}}{d t}=A J_{c}-I(t)
$$

where $A$ is the electrode area, $J_{c}$ is the net charged particle conduction current, and $I(t)$ is the current flowing in the external circuit. The voltage across the bias capacitor is related to the charge on the capacitor from $V_{c}=Q_{c} / C$. By then discretising Eq. (2), using Eq. (1), and making use of Gauss's law at the electrode boundary to establish a relationship for the surface charge density (see Ref. 31 for more details), an expression can be obtained for the unknown electrode potential $\phi_{0}$, which is then solved at each time step.

\section{CODE VALIDATION}

Before simulating the system in Fig. 1, two tests cases are run to benchmark the code and confirm that it is

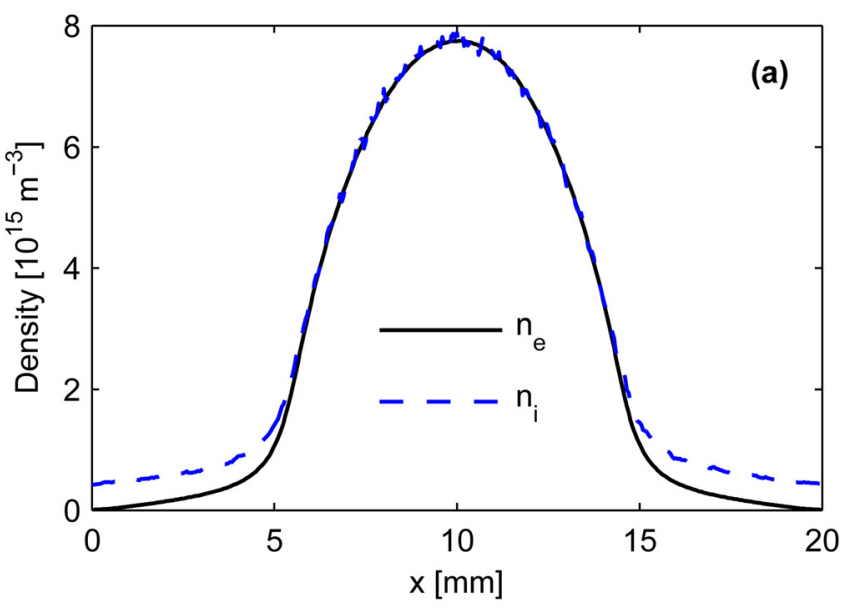

(b)

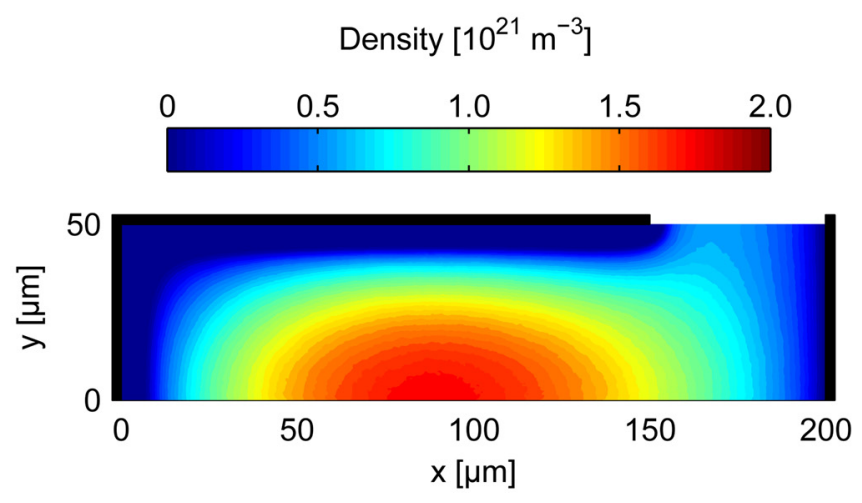

FIG. 2. (Color online) (a) Time-averaged equilibrium profiles of the ion density ( $n_{i}$, dashed line) and electron density $\left(n_{e}\right.$, solid line), for a $100 \mathrm{mTorr}$ parallel plate CCP discharge (test case A). (b) Contour plot of the equilibrium plasma density of a dc micro-HC discharge at 10 Torr (test case $\mathrm{B}$ ).

operating correctly. In the first test (test case A), we make use of a parallel plate capacitively coupled system, with electrodes spaced $2 \mathrm{~cm}$ apart, and a background pressure of 100 mTorr. Since this effectively represents a one-dimensional systems, the transverse, $y$, direction is set to a small value so that fewer grid points and particles are needed. The time step is set to $3.5 \times 10^{-11} \mathrm{sec}$, and about $6 \times 10^{4}$ electron-ion pairs are used, each representing $5 \times 10^{5}$ real particles. The rf voltage amplitude used is $270 \mathrm{~V}$ and secondary electron emission is turned off. The above test parameters were chosen due to the presence of other PIC data within the literature for comparison, such as that in Ref. 32. Figure 2(a) shows the time-averaged plasma density obtained after a steady state was reached in the simulation. The results are seen to agree very well with those presented in Fig. 3 of Ref. 32. Additionally, the dc self bias is found to be $0 \mathrm{~V}$, as expected due to the symmetrical nature of the discharge.

In the second test (test case B), we simulate a similar system to the dc micro-HC in Ref. 20, with the only difference being that our simulation uses a Cartesian slab geometry as opposed to the cylindrical geometry in Ref. 20. The hole/slit half-width is $50 \mu \mathrm{m}$, with a depth of $150 \mu \mathrm{m}$. The total simulation length is $200 \mu \mathrm{m}$, thus resulting in a gap 


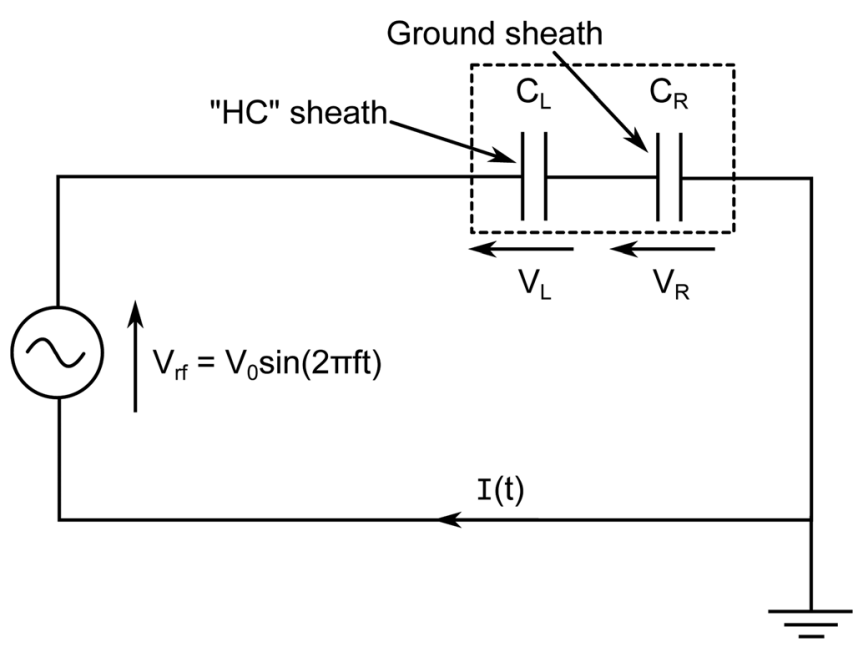

FIG. 3. Capacitive voltage divider formed by the plasma sheaths at the HC and ground electrodes.

length of $50 \mu \mathrm{m}$ between the anode and cathode. The time step is around $1 \times 10^{-13} \mathrm{sec}$ and several hundreds of thousands of electron-ion pairs are used, each representing $2.5 \times 10^{7}$ real particles. Only secondary electron emission due to ion bombardment is present, and the background gas pressure is 10 Torr. A current source is used within the external circuit (with the bias capacitor removed), using a current density matching that used in Ref. 20. Figure 2(b) shows a contour plot of the time-averaged plasma density obtained after a steady state was reached. These results are in good agreement with those in Fig. 2 of Ref. 20. Slight differences can be attributed to the use of a Cartesian slab geometry here as opposed to the cylindrical coordinate system used in Ref. 20. At the large densities obtained $\left(\sim 10^{21} \mathrm{~m}^{-3}\right)$, the mean-free path for electron-ion Coulomb collisions ${ }^{4}$ is of the order of about $100 \mu \mathrm{m}$ (i.e., similar to the system dimensions). This suggests that these collisions could be important in micro-HC discharges. However, since these collisions were not simulated in Ref. 20, we have not included them in the present benchmarking comparison. At the much lower densities $\left(10^{16}-10^{17} \mathrm{~m}^{-3}\right)$ to be simulated below, the mean-free path is of the order of $1 \mathrm{~m}$, which is much larger than the system dimensions. Hence, Coulomb collisions are neglected in the present investigation.

As a final test, with the plasma turned off, and using a parallel plate geometry, the system capacitance can be easily calculated, and from an ac circuit analysis, the voltage drop across the bias and 'simulation' capacitors can be analytically calculated. These values were found to match those obtained from the simulation.

\section{RESULTS}

\section{A. Capacitive divider}

Initial simulations were run without the bias capacitor $\left(C_{B}\right)$ and with a much smaller gap length of only a few millimeters between the electrodes. Under these conditions, a stable discharge could be obtained within the slit for pressures between 100 and 500 mTorr with applied voltages of around $V_{0}=400 \mathrm{~V}$ and evidence of high energy electrons oscillating between opposite walls of the slit was seen. Observation of the system current-voltage characteristics, however, showed that a small dc current was present in the external circuit. To prevent this current, and more accurately model real experimental systems, the bias capacitor was added to the external circuit. However, with this bias capacitor present a large positive self bias was produced, and a discharge could no longer be obtained within the slit. By observing the time-averaged plasma potential, it was found that the voltage drop across the powered electrode was very small compared with that across the ground electrode and, thus, a discharge could not be sustained in the slit.

The above problem is related to the well-known capacitive voltage divider effect of the sheaths at each electrode in CCP discharges and is not present in dc systems. To illustrate this voltage divider, we consider the simplified plasma circuit model shown in Fig. 3. Here, each of the electrode sheaths is treated as a parallel plate capacitor with capacitances $C_{L}$ and $C_{R}$, respectively. In this case, the capacitance can be obtained from

$$
C_{i}=\frac{\epsilon_{0} A_{i}}{d_{i}},
$$

where $\epsilon_{0}$ is the permittivity of free space, $A_{i}$ is the area of the capacitor, and $d_{i}$ is the distance between the plates of the capacitor. Thus, we see that the capacitance is proportional to the electrode area, and if for sake of argument, we assume that $d_{L} \approx d_{R}$, we see that the electrode with the greater area has a larger capacitance. The complex impedance of a capacitor is given by $Z=-j /(2 \pi f C)$ (where $\left.j^{2}=-1\right)$, and thus a smaller capacitance results in a larger impedance. By using Kirchhoff's current and voltage laws, we can show that the voltage drop across the left-hand sheath capacitor (the "HC" electrode) is given by

$$
\left|\frac{V_{L}}{V_{r f}}\right|=\frac{A_{R}}{A_{R}+A_{L}},
$$

where $A_{L}$ and $A_{R}$ are the areas of the left-hand and righthand electrodes, respectively. With a system geometry such as that of configuration 1 in Fig. 1(a), the ground electrode area is significantly smaller than that of the powered electrode, and thus, only a small voltage is dropped across the powered electrode. If this voltage is too small, then a HCE cannot be initiated, and a plasma cannot be sustained within the slit. In most experimental systems, the ground electrode area is typically much larger than the powered electrode area, since the reactor walls are usually grounded as well. Thus, most of the applied voltage is dropped across the powered electrode. Experimental work with rf HCs in Ref. 33 shows that breakdown in HC systems can in some cases occur easier for smaller area (when compared with the ground area) powered electrodes and that deposition of an insulating layer on the grounded reactor walls (which would affect its capacitance) influences both the plasma potential and the dc self bias that develops.

In a simulation context, however, the electrode areas produce significant problems, since the reactor walls are 
usually not simulated due to the severe increase in run times and computational complexity. This problem is also not encountered in simulations of dc systems, such as in Ref. 20, where capacitive effects are negligible, and thus is only a problem when simulating high-frequency systems. To overcome this problem within the simulation, configuration 2 in Fig. 1(a) is used, where a larger ground plate has been added to the top of the simulation domain. However, in order to obtain a sufficiently large ground area from this extra plate, a larger gap is needed compared to the dc simulations, hence the choice of a distance of $40 \mathrm{~mm}$ (which also matches the gap length in Ref. 10). With these changes, the self bias that forms is now either negative or close to zero (the larger plasma density in the slit changes the sheath width $d$, and so the analysis above is more complicated in reality ${ }^{29}$ ), and the voltage drop across the powered electrode is now sufficiently large to produce a discharge within the slit. In order to prevent a HCE forming within the new grounded electrode slit geometry, secondary electron emission is turned off on all ground surfaces. Since the grounded electrode area in experimental systems is usually much larger than the powered electrode area, the voltage drop across the sheath at the grounded electrode is usually quite small (of the order of a few tens of volts $\left.{ }^{29}\right)$. Thus, any secondary electrons emitted from grounded surfaces are expected to have only a small effect.

\section{B. Initial characterization}

With the changes made above in Sec. IV A, stable hollow cathode discharges could be produced over a range of pressures and applied voltages. Using a representative pressure of 258 mTorr (within the range of values used in Ref. 10) and an applied voltage of $400 \mathrm{~V}$, we explicitly test the importance of secondary electron emission within the discharge. This is demonstrated in Fig. 4, which shows the time-averaged plasma potential (Fig. 4(a)) and plasma density (Fig. 4(b)) after $10 \mu$ s for a number of test cases. Case 1 is a simple plane powered electrode (here the hollow cathode geometry has been removed from configuration 2 in Fig. 1(a)), with an electron produced secondary electron emission coefficient, $\gamma_{e}$, of 0 and an ion produced secondary electron emission coefficient, $\gamma_{i}$, of 0.2 ; case 2 is a slit electrode with $\gamma_{e}=0$ and $\gamma_{i}=0$; case 3 is a slit electrode with $\gamma_{e}=0.2$ and $\gamma_{i}=0$; and case 4 is a slit electrode with $\gamma_{e}=0$ and $\gamma_{i}=0.2$. For clarity, we only plot the axial distance from $x=0 \mathrm{~mm}$ to $x=18 \mathrm{~mm}$.

Case 1 shows that a large negative self bias forms at the powered electrode, as expected due to the larger ground area. Cases 2 and 3 show that the plasma does not penetrate very far into the slit and is present just near the exit. These cases also show a negative self bias. The peak density for these cases is slightly higher than that for case 1, suggesting that the presence of the slit enhances the discharge somewhat. Cases 2 and 3 are very similar, this despite $\gamma_{e}=0.2$ for case 3, and will be discussed further in Sec. V. Case 4 shows very different behaviour, where now the plasma has penetrated deep within the slit and has a significantly higher density than the other cases, peaking at about $x=5 \mathrm{~mm}$ with a value of $3 \times 10^{17} \mathrm{~m}^{-3}$. The plasma potential is also much
Potential

(a)
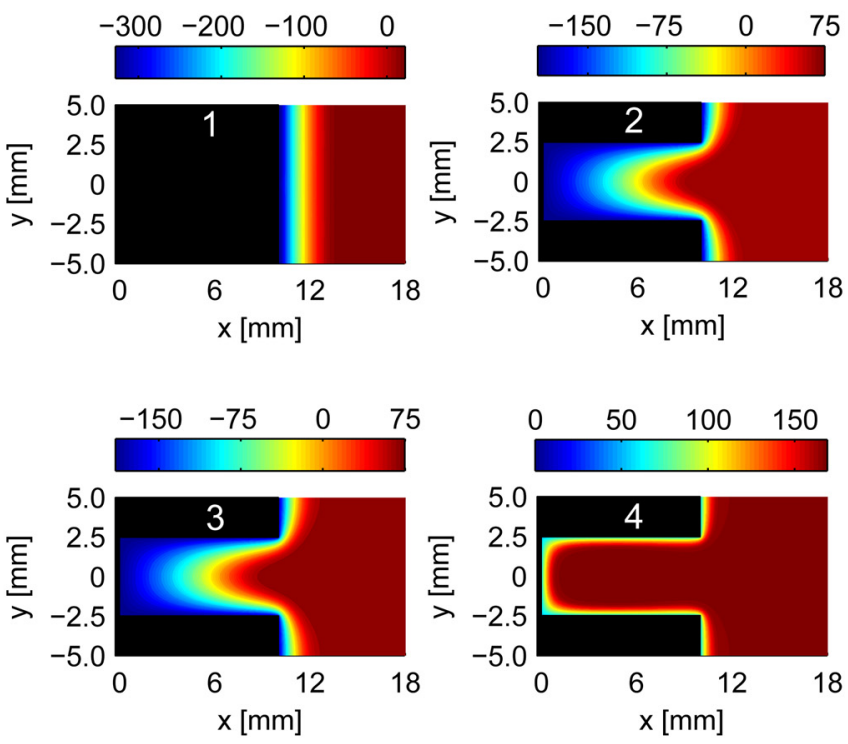

Density, $\mathrm{n}_{\mathrm{e}}$

(b)
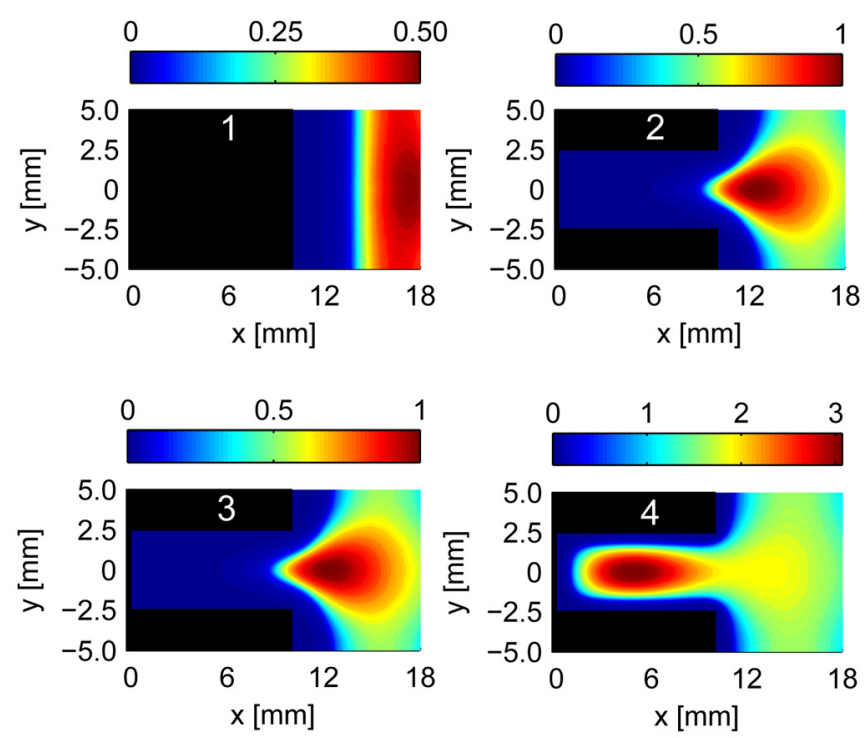

FIG. 4. (Color online) Contour plots of the time-averaged (a) plasma potential and (b) plasma density, for a number of test cases; plane powered electrode with $\gamma_{e}=0$ and $\gamma_{i}=0.2$ (case 1); slit powered electrode with $\gamma_{e}=0$ and $\gamma_{i}=0$ (case 2); slit powered electrode with $\gamma_{e}=0.2$ and $\gamma_{i}=0$ (case 3); and slit powered electrode with $\gamma_{e}=0$ and $\gamma_{i}=0.2$ (case 4). The color bars have units of [V] for (a) and units of $\left[10^{17} \mathrm{~m}^{-3}\right]$ for (b). The black regions in (a)-(b) show the geometry and location of the powered electrode for each case. To aid clarity, the results in (a)-(b) have been mirrored about the symmetry plane $y=0 \mathrm{~mm}$.

higher, and the electrode has a small positive dc bias, despite the larger ground area. This can be attributed to the much larger plasma density within the slit, and consequently, the smaller sheath width, which results in a larger capacitance and, hence, a larger effective electrode area. After $10 \mu$ s of simulation time, $\gamma_{i}$ was set to 0 for case 4 , and after an additional $5 \mu \mathrm{s}$, the plasma density had decreased, and the plasma had almost completely left the slit. In addition, the self bias now became negative. 
In the experiments in Ref. 10, it was found that by using different materials (which have different secondary electron emission coefficients) for the powered electrodes, the density could be enhanced to a larger or smaller extent. ${ }^{30}$ As a test of this, simulations were run with lower secondary electron emission coefficients of $\gamma_{i}=0.1$ and $\gamma_{i}=0.05$ (with $\gamma_{e}=0$ for both cases). For larger values of $\gamma_{i}$, the simulation density was observed to be higher, and as the coefficient was decreased, the plasma would not penetrate as deep into the slit for the same initial conditions. For the plasma to penetrate the slit substantially (more than about 50\%), a secondary electron emission coefficient of about 0.1 was needed.

Although a given rf voltage is set for the voltage source in Fig. 1(b), due to the density that evolves within/near the slit, the voltage drop across the powered electrode is not known a priori. Fortunately though, the experimental data in Ref. 10 are plotted as a function of the time-averaged voltage difference at the powered electrode, and so data exists for comparison. Such a comparison, while useful, must be taken carefully, since here we have used a multi-slit electrode instead of a multi-hole electrode as used in Ref. 10, and the extra grounded electrode piece of configuration 2 in Fig. 1(a) is absent in the experiment. In addition, due to the long simulation times needed because of the system size and number of particles, the simulations have not fully converged yet, although we believe they have been run long enough for all essential physics to be present. The experimental and simulation results are shown in Fig. 5, where the simulation results for the slit (closed circles) are seen to be in fairly reasonable agreement with the experimental densities ${ }^{10}$ for a hole diameter of $5 \mathrm{~mm}$ over the range of values tested. The simulation results for the plane electrode (closed squares), however, are significantly lower, suggesting that the additional grounded simulation piece disrupts the plane electrode system to a

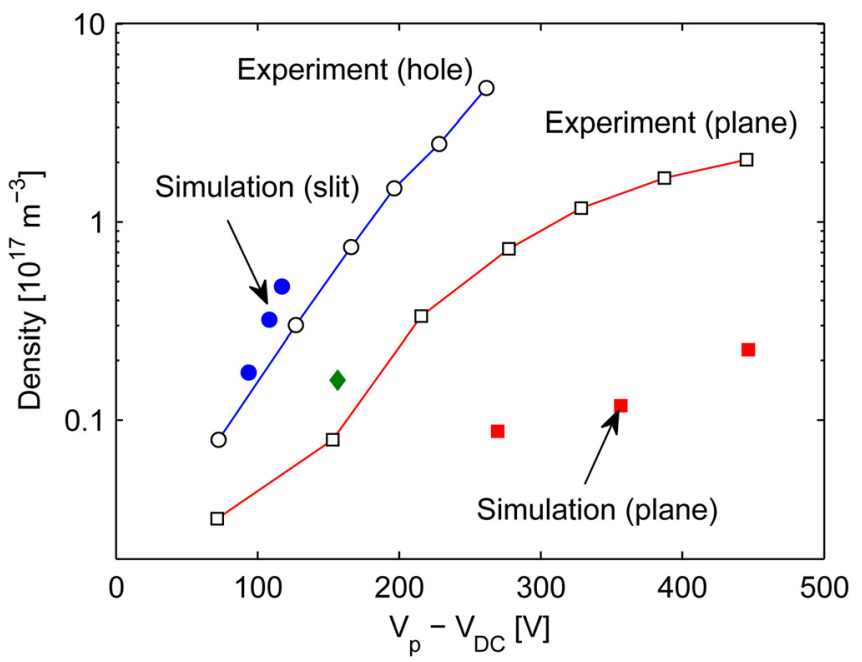

FIG. 5. (Color online) Plasma density within the center of the discharge $(x=30 \mathrm{~mm}$ and $y=0 \mathrm{~mm})$ as a function of the voltage difference between the time-averaged plasma potential and the self bias at the powered electrode. The argon gas pressure is 258 mTorr. The experimental results are taken from Fig. 3(c) in Ref. 10, with results for a plane electrode (open squares) and a multi-hole electrode (with a hole diameter of $5 \mathrm{~mm}$; open circles). The simulation results are time-averaged, obtained after $10 \mu \mathrm{s}$. The closed diamond shows the simulation results for a plane powered electrode with the extra ground piece of configuration 2 in Fig. 1(a) removed. larger extent. To test this, the extra ground piece was removed, and an additional simulation was run. These results are shown as the closed diamond, which is seen to be in reasonable agreement with the experimental results. Pressures of between 100 and 500 mTorr were tested over a range of applied voltages $(200-500 \mathrm{~V})$ for case 4, although at 100 mTorr a plasma was not present within the slit yet.

The above results all suggest that the observed density enhancement in Ref. 10 is, as hypothesised by those authors, a result of a $\mathrm{HC}$ discharge resulting from secondary electron emission due to ion bombardment. As noted above, in the absence of secondary electrons, a discharge could not be initiated or sustained in the simulation. This suggests that $\mathrm{rf}$ sheath heating is not a strong enough power deposition mechanism in the present case. To understand and justify this conclusion further, we look now at the ionization and power absorption characteristics in detail.

\section{Ionization characteristics}

Figure 6 shows a contour plot of the spatial ionization rate within the $\mathrm{HC}$ for case 4 , averaged over a number of $\mathrm{rf}$ periods. As seen, the ionization rate is peaked in the center of the slit and roughly follows the shape of the plasma density profile in Fig. 4(b), with a maximum value of about $1 \times 10^{23} \mathrm{~m}^{-3} \mathrm{~s}^{-1}$. Further insight into the ionization behaviour can be obtained by observing the temporal variation of the ionization rate. Here, we compare the behaviour of cases 1 and 4 of Fig. 4. Case 1 is the plane powered electrode case, where although secondary electron emission due to ion bombardment is present, no HCE can form due to the absent of the slit. A HCE was observed for case 4, which has a slit geometry and secondary electron emission.

The temporal variation of the ionization rate (averaged over the whole simulation domain) is shown in Fig. 7 for cases 1 (part (a)) and 4 (part (b)) of Fig. 4. The thick solid line shows the electrode voltage with rf phase, demonstrating
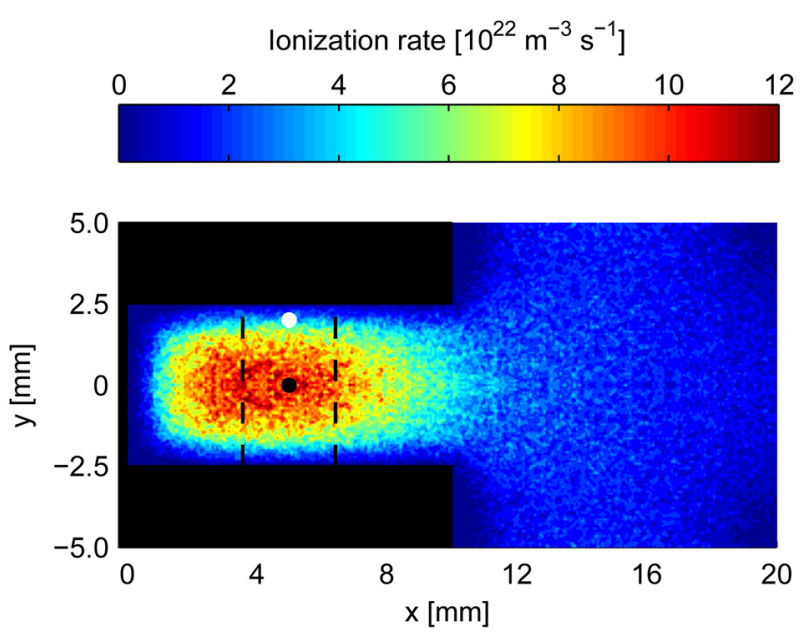

FIG. 6. (Color online) Contour plot of the time-averaged (over $10 \mathrm{rf}$ periods) spatial ionization rate. To aid clarity, the results have been mirrored about the symmetry plane $y=0 \mathrm{~mm}$. The black and white circles mark locations at which the electron energy probability function (EEPF) is calculated (see Sec. IV E), while the vertical dotted lines mark the boundary of the region used to find the spatio-temporal ionization and power absorption profiles (see Secs. IV C and IV D). 

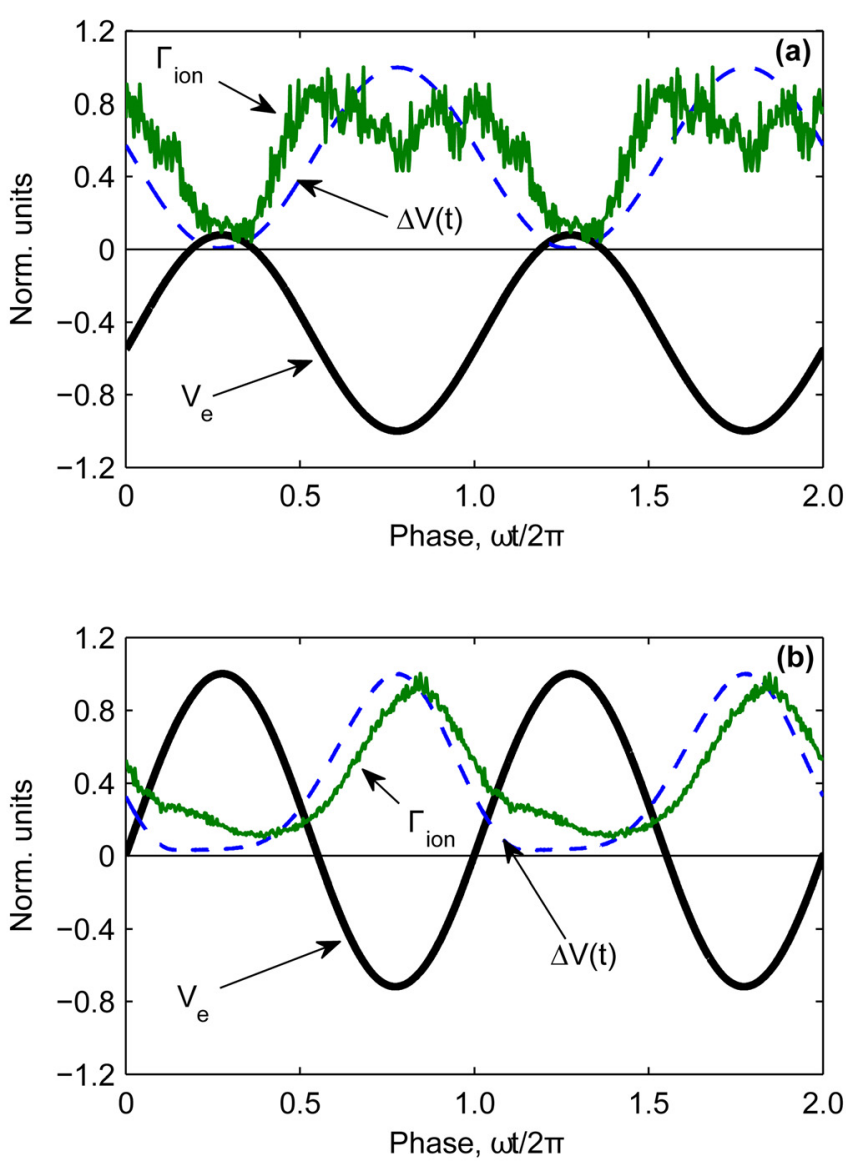

FIG. 7. (Color online) Normalized plots of the electrode voltage $\left(V_{e}\right.$; thick solid line), total ionization rate $\left(\Gamma_{i o n}\right.$; thin solid line), and time-varying voltage difference between the plasma potential in the center of the $\mathrm{HC}$ (at $x=5 \mathrm{~mm}$ and $y=0 \mathrm{~mm}$ ) and the electrode voltage $(\Delta V(t)$; dashed line) as a function of rf phase, for (a) case 1, and (b) case 4, in Fig. 4. To aid clarity, the results have been plotted for $2 \mathrm{rf}$ periods.

the large negative self bias for case 1, while the thin solid line shows the globally averaged ionization rate. Also plotted in Fig. $7(\mathrm{a})$ is the time-varying voltage difference $(\Delta V)$ between the plasma potential and the electrode voltage. As is seen, the ionization rate peaks between $\omega t / 2 \pi=0.4-0.6$, at the location where $\Delta V$ is rising, and is correlated with the expansion of the sheath into the plasma. This behaviour has been observed in a number of other PIC simulations of CCP discharges ${ }^{34,35}$ and is characteristic of a sheath heated discharge. A second peak is seen at around $\omega t / 2 \pi=1$, which is a result of the secondary electrons accelerated across the fully expanded sheath.

By contrast, for case 4 in Fig. 7(b), the electrode voltage has a small positive dc bias, and the ionization rate peaks after the maximum $\Delta V$ once the sheath has fully expanded and when in fact it is beginning to collapse. This is characteristic of secondary electron heated discharges ${ }^{35}$ and represents the secondary electrons and the additional electrons produced through ionization, travelling into the plasma. No significant ionization is observed as the sheath is expanding. These above observations are consistent with the results in Sec. IV B and suggest that the $\mathrm{HC}$ discharge is a result of secondary electron heating.

Figure 8 shows a spatio-temporal plot of the distribution of ionization events for the plane electrode simulation of

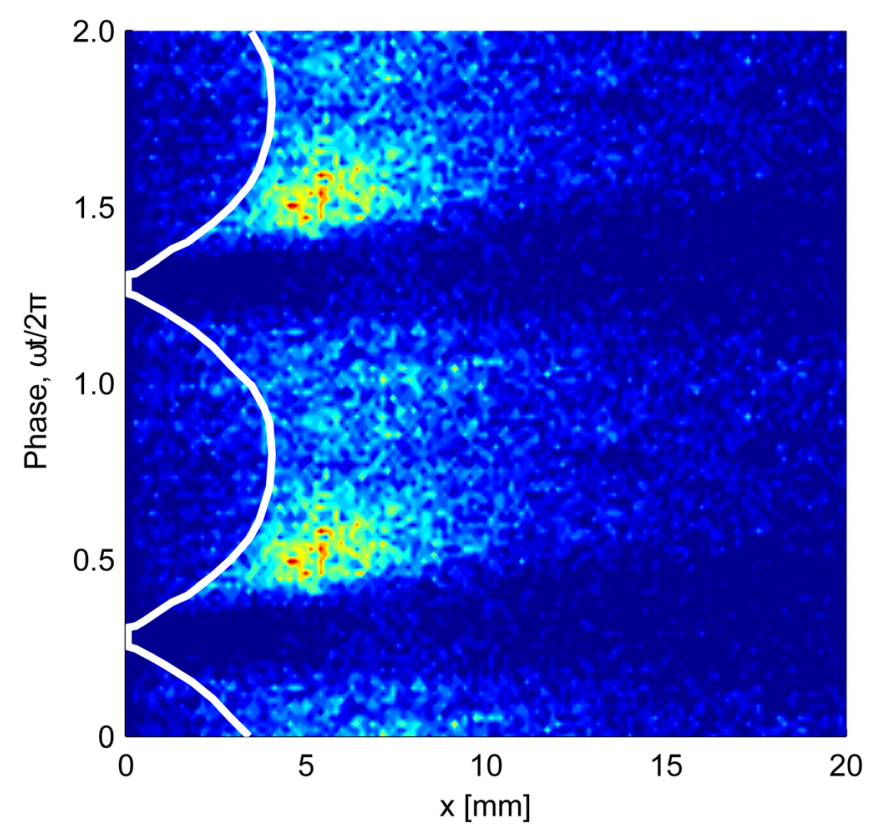

FIG. 8. (Color online) Spatio-temporal distribution of the ionization rate for case 1 in Fig. 4 . The results are averaged over $10 \mathrm{rf}$ periods and plotted using the axial dimension $(x)$. The spatial results have been averaged over the lateral dimension $(y)$. To aid clarity, the results have been plotted for $2 \mathrm{rf}$ periods. The white solid line shows the time-varying sheath width throughout the rf period. The color plot is normalized such that dark regions correspond to 0 , and light regions to 1 .

case 1 , collected over $10 \mathrm{rf}$ periods using the axial coordinate $(x)$ as the spatial dimension. The white solid line shows the instantaneous position of the sheath, which is obtained by finding where $\partial^{2} \phi / \partial x^{2}$ first becomes zero (here $\phi=\phi(x, y)$ is the potential). As seen, the ionization is most intense at the sheath edge, during the phase when the sheath is expanding into the plasma, and this ionization propagates away from the sheath edge into the bulk plasma. This behaviour is again characteristic of sheath heated discharges and has been observed in a number of other simulations of standard CCP systems. ${ }^{34,35}$ Some ionization is present within the sheath due to the presence of secondary electrons. This behaviour is in stark contrast to that for case 4, which is shown in Fig. 9. This again shows the distribution of ionization events collected over $10 \mathrm{rf}$ periods, using the transverse coordinate $y$ as the spatial dimension. Again the white solid lines indicate the instantaneous sheath position. Most of the ionization is seen to occur within the center of the slit, at an rf phase close to where the sheath has fully expanded. Almost no ionization is observed as the sheath begins to expand or once the sheath has fully collapsed. Also, there seems to be no evidence for a propagating ionization front, such as that in Fig. 8. Note that the spatio-temporal distributions presented in Figs. 8 and 9 are very similar to those in Figs. 10 and 11 of Ref. 35 for secondary electron and sheath heated CCP discharges.

\section{Power absorption characteristics}

In order to directly observe the power absorption process, we use a spatio-temporal plot of the electron power absorption $\left(\mathbf{E} \cdot \mathbf{J}_{e}\right.$, where $\mathbf{J}_{e}$ is the electron current density) averaged over $10 \mathrm{rf}$ periods within the slit, as shown in Fig. 10 for case 4. 


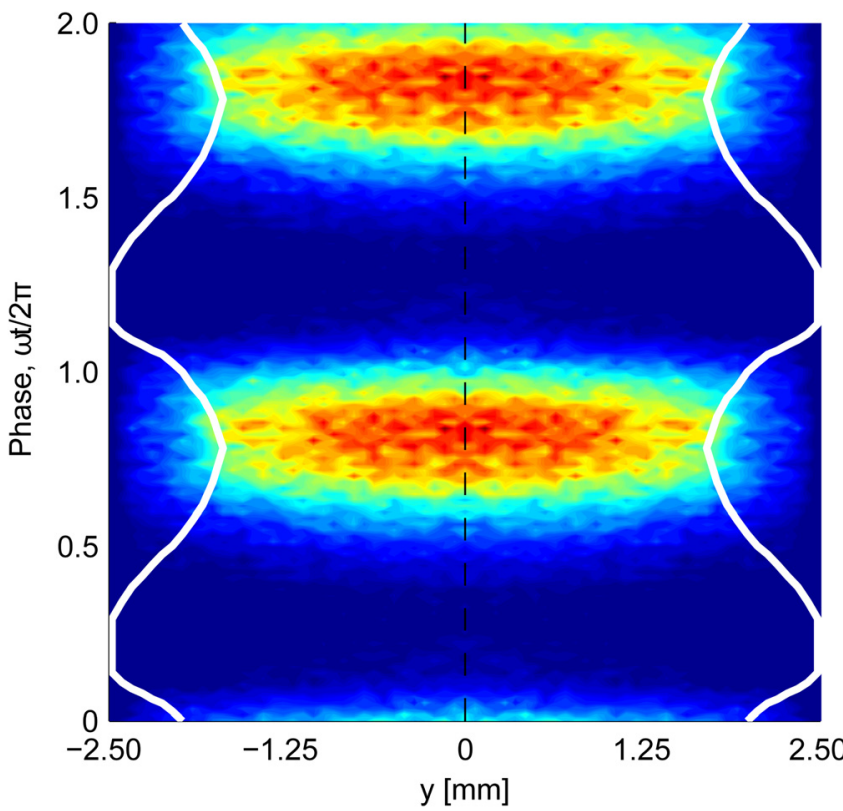

FIG. 9. (Color online) Spatio-temporal distribution of the ionization rate for case 4 in Fig. 4. The results are averaged over $10 \mathrm{rf}$ periods and plotted using the lateral slit dimension $(y)$. The spatial results have been averaged over the axial dimension $(x)$ for values between the vertical dashed lines in Fig. 6. To aid clarity, the results have been mirrored about the symmetry plane $y=0 \mathrm{~mm}$ (shown as the vertical dashed line) and plotted for $2 \mathrm{rf}$ periods. The white solid lines show the time-varying sheath width throughout the rf period. The color plot is normalized such that dark regions correspond to 0 , and light regions to 1 .

Again the thick dashed line shows the instantaneous sheath position. The red regions (e.g. regions 1 and 3 in Fig. 10) indicate positive power transfer, while the blue regions (e.g. region 2 in Fig. 10) indicate negative power transfer. The power absorption occurs at or within the sheath, and as is seen, there are two main regions of positive power transfer: region 1 within the sheath, which indicates the power transfer that occurs in accelerating the secondary electrons, and region 3 , which indicates power absorption near the sheath edge as the sheath expands into the plasma. Region 2 shows negative power absorption as the sheath collapses. Although it was argued in Secs. IV B and IV C that the discharge for case 4 is a secondary electron heated discharge, we see that there is some evidence of sheath heating present. However, the integrated total power absorption in region 3 is found to be almost exactly equal to the integrated negative power absorption in region 2 . Thus, the net effect of sheath heating is 0 . By contrast, the integrated power absorption of region 1 is significantly greater than that for region 3 and accounts for almost all of the electron heating. This explains why the discharge within the slit was observed to die out when the secondary electron coefficient was set to zero for case 4 in Sec. IV B. By removing the effect of region 1 in Fig. 10, the net power absorption in regions 2 and 3 is 0 .

The power absorption found for case 4 is very different to that in Fig. 11, which shows a spatio-temporal plot of the electron power absorption for case 1 . Here, electron heating within the sheath is less pronounced than that at the sheath edge and than that in Fig. 10. By using a similar region demarcation as in Fig. 10, the positive power absorption in region 3 is now found to be larger than that in region 2, so

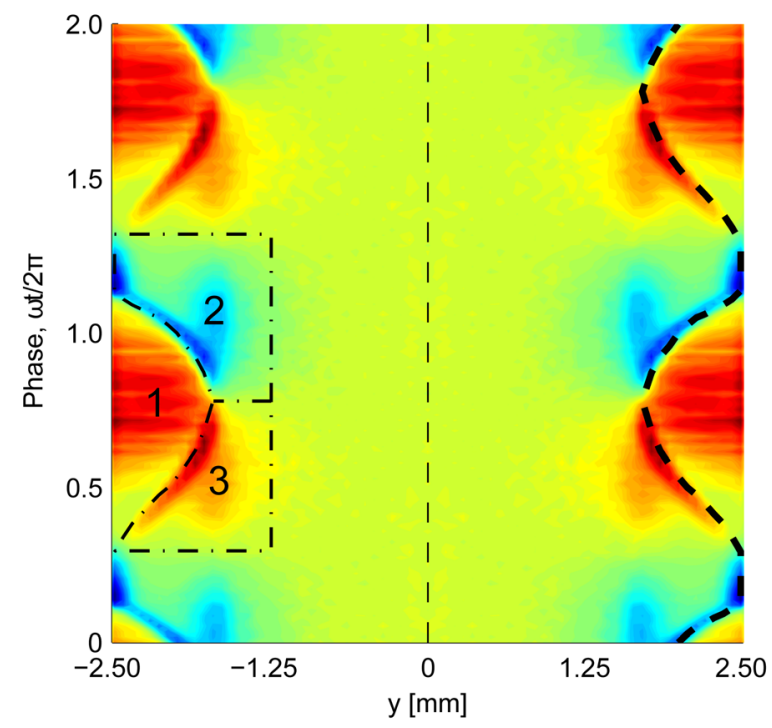

FIG. 10. (Color online) Spatio-temporal distribution of the total electron power absorption rate $\left(\mathbf{E} \cdot \mathbf{J}_{e}\right)$ for case 4 in Fig. 4 . The results are averaged over $10 \mathrm{rf}$ periods and plotted using the lateral slit dimension $(y)$. The spatial results have been averaged over the axial dimension $(x)$ for values between the vertical dashed lines in Fig. 6. To aid clarity, the results have been mirrored about the symmetry plane $y=0 \mathrm{~mm}$ (shown as the vertical dashed line) and plotted for $2 \mathrm{rf}$ periods. The thick dashed line shows the timevarying sheath width throughout the rf period. Power absorption within and near the sheath is indicated by the dashed regions marked 1-3 (see Sec. IV D). The color plot is normalized such that dark blue (e.g. region 2) is -1 , light regions are 0 , and bright red (e.g. regions 1 and 3 ) is 1 .

that even in the absence of secondary electron emission, net power absorption can occur. Thus, sheath heating does contribute to the electron heating process in this case, and as seen in Figs. 7 and 8, this results in very different ionization behaviour to case 4 .

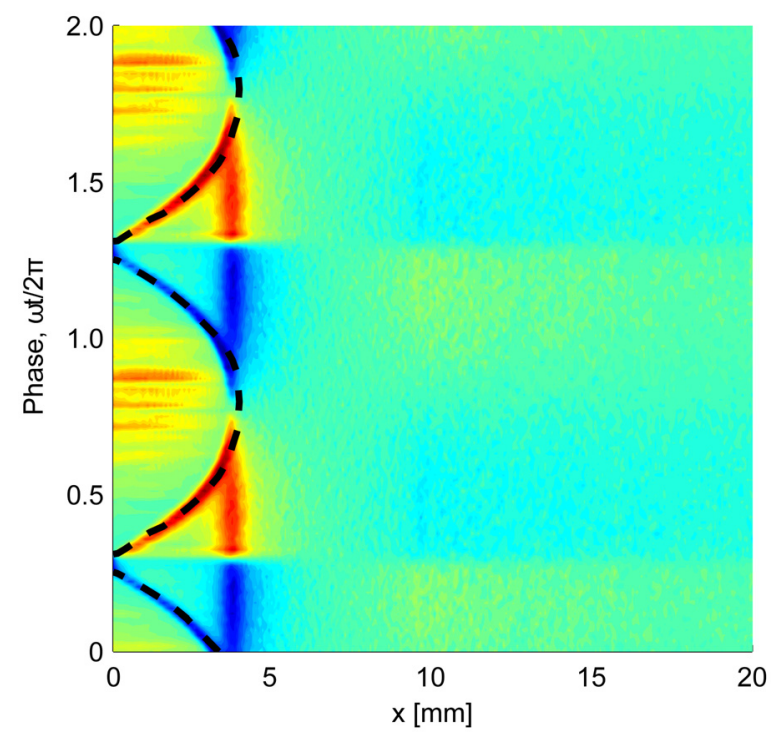

FIG. 11. (Color online) Spatio-temporal distribution of the total electron power absorption rate $\left(\mathbf{E} \cdot \mathbf{J}_{e}\right)$ for case 1 in Fig. 4 . The results are averaged over $10 \mathrm{rf}$ periods and plotted using the axial dimension $(x)$. The spatial results have been averaged over the lateral dimension $(y)$. To aid clarity, the results have been plotted for $2 \mathrm{rf}$ periods. The thick dashed line shows the time-varying sheath width throughout the rf period. The color plot is normalized such that dark blue (e.g. the corresponding region 2 in Fig. 10) is -1 , light regions are 0, and bright red (e.g. the corresponding regions 1 and 3 in Fig. 10) is 1. 


\section{E. Electron dynamics}

To gain further insight into the electron dynamics, we study the electron distribution function, shown in Fig. 12, and its evolution through an rf cycle, for case 4 . Here, the color plot makes use of a logarithmic scale to clearly distinguish the high energy electrons. The low energy electrons are represented by the dark bar centered around zero energy. The positive and negative energies represent electrons moving to the right and left ( $x$ direction), respectively, in part (a) or up and down ( $y$ direction) in part (b). The distribution function shows significant modulation of the high energy electrons through the rf phase, with regions absent of high energy electrons, followed by regions with a significant high energy electron presence. The dashed line on the positive portion of the graph in Fig. 12(a) (representing electrons moving to the right) is the time-varying voltage difference, $\Delta V$, which is seen to fit the limits of the high energy electron population very well. In fact, this is the potential (across the sheath at the back wall of the slit located at $x=0 \mathrm{~mm}$ ) that
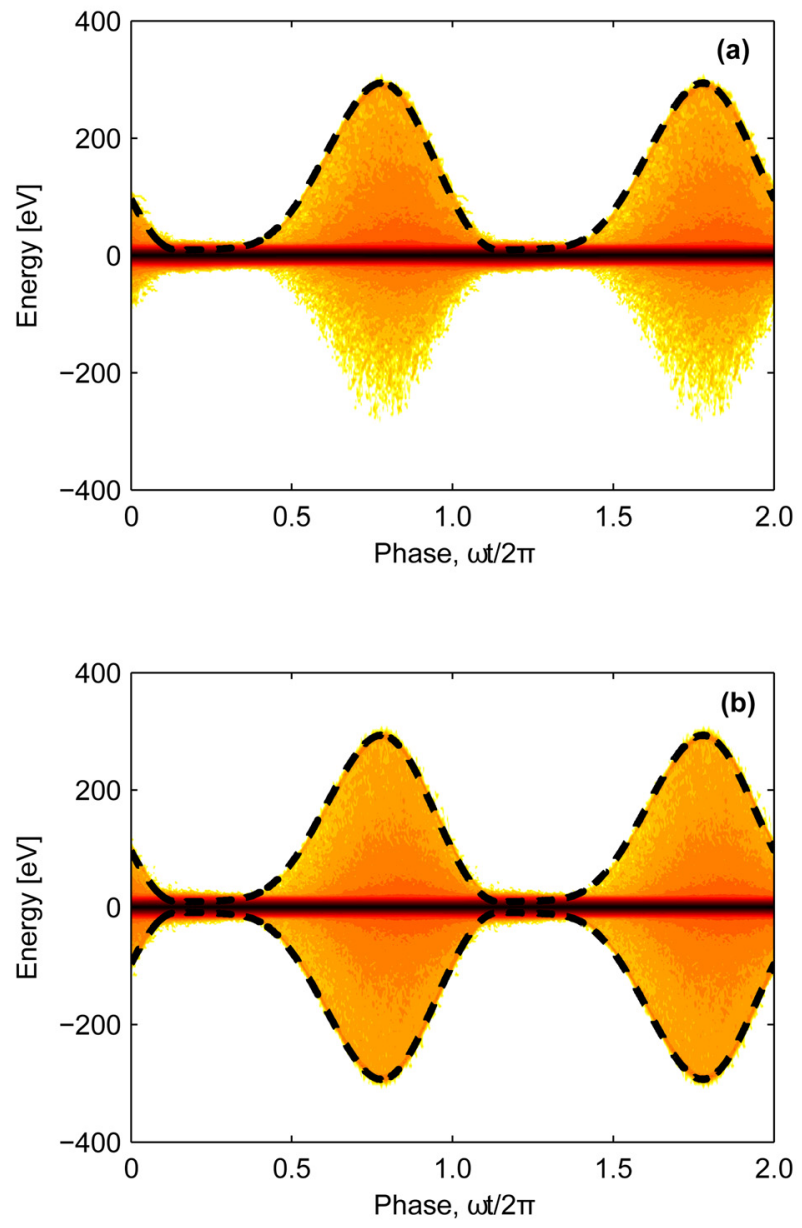

FIG. 12. (Color online) Contour plots of the electron velocity distribution function in (a) the $x$ direction and (b) $y$ direction, averaged over the entire simulation domain, as a function of rf phase, for case 4 in Fig. 4. To aid clarity, the results have been plotted over $2 \mathrm{rf}$ periods. The distribution functions in (a)-(b) have been normalized and are plotted using a logarithmic color scale, such that dark regions correspond to 0 , and white $\rightarrow-\infty$. The thick dashed lines in (a)-(b) show the time-varying voltage difference $(\Delta V(t))$ between the plasma potential in the center of the $\mathrm{HC}$ (at $x=5 \mathrm{~mm}$ and $y=0 \mathrm{~mm}$ ) and the electrode voltage as a function of rf phase. accelerates the secondary electrons to their respective final energies. This modulation is unique to rf discharges, since in a dc $\mathrm{HC}$, secondary electrons are accelerated to essentially the same energy across the constant sheath potential. Although not obvious from the figure, the electrons do not instantaneously follow $\Delta V$, and there is a small time delay of about $0.1 \mathrm{~ns}$; equal to the time needed to accelerate the electrons across the sheath to their final energy.

The left moving electrons in Fig. 12(a) (negative energies), while showing similar behaviour, are less populated in the high energy regions. This is a consequence of the absence of secondary electron emission on the right hand boundary (the ground wall), which was turned off to prevent a HC discharge in this region. The presence of high energy secondaries travelling in this direction is due to electronneutral collisions which scatter electrons into this direction. Figure 12(b) shows similar behaviour to 12(a), but now due to the symmetry of the $\mathrm{HC}$ slit, both sides of the distribution are virtually identical. These high energy electrons are mostly those heated by region 1 in Fig. 10, and as expected, the highest energy electrons occur at the phase where the sheath has fully expanded, and hence where the voltage difference between the electrode and plasma is the greatest.

These high energy electrons can also be seen in the electron energy probability function (EEPF), shown in Fig. 13(a), averaged over a number of rf periods. The solid line shows the EEPF at the center of the slit at $x=5 \mathrm{~mm}$ and $y=0 \mathrm{~mm}$ (closed circle in Fig. 6), while the dashed line shows the EEPF near the sheath edge at $x=5 \mathrm{~mm}$ and $y=2.25 \mathrm{~mm}$ (open circle in Fig. 6). The high energy electron population extends from about $50 \mathrm{eV}$ to just over $300 \mathrm{eV}$, close to the maximum voltage difference between the plasma and electrode. The inset figure shows a magnified view of the low energy electron region between 0 and $50 \mathrm{eV}$, where two electron populations can be seen, with temperatures of about $T_{e_{1}}=3 \mathrm{eV}$ and $T_{e_{2}}=1.75 \mathrm{eV}$, respectively.

An example trajectory of a secondary electron emitted from one of the lateral slit walls is shown in Fig. 13(b). The electron is followed for a total time of $7.5 \mathrm{~ns}$ (about $10 \%$ of an rf period), and the arrows indicate the direction of motion. The closed circles mark an electron-neutral collision event (e.g. elastic, excitation and ionization collisions). The pendular motion of the secondary electron is clearly seen by the 8 distinct bounces (helped by a number of collisions) it makes between the walls throughout its trajectory.

\section{F. Current-voltage characteristics}

Figure 14(a) shows the current-voltage characteristic for case 4 over $2 \mathrm{rf}$ periods. The electrode voltage (thick solid curve) oscillates sinusoidally from $-280 \mathrm{~V}$ to $390 \mathrm{~V}$ and has a small dc bias of about $55 \mathrm{~V}$. The central plasma potential (obtained at $x=5 \mathrm{~mm}$ and $y=0 \mathrm{~mm}$; dashed curve) is seen to follow the positive portion of the electrode voltage but is close to 0 during the negative portion. Also plotted in Fig. 14(a) is the total current (light solid curve) in the system, which varies approximately sinusoidally, and has an amplitude of $2.5 \mathrm{Am}^{-1}$. The current is seen to lead the voltage by a phase angle of almost $\pi / 2$, and so the discharge is 

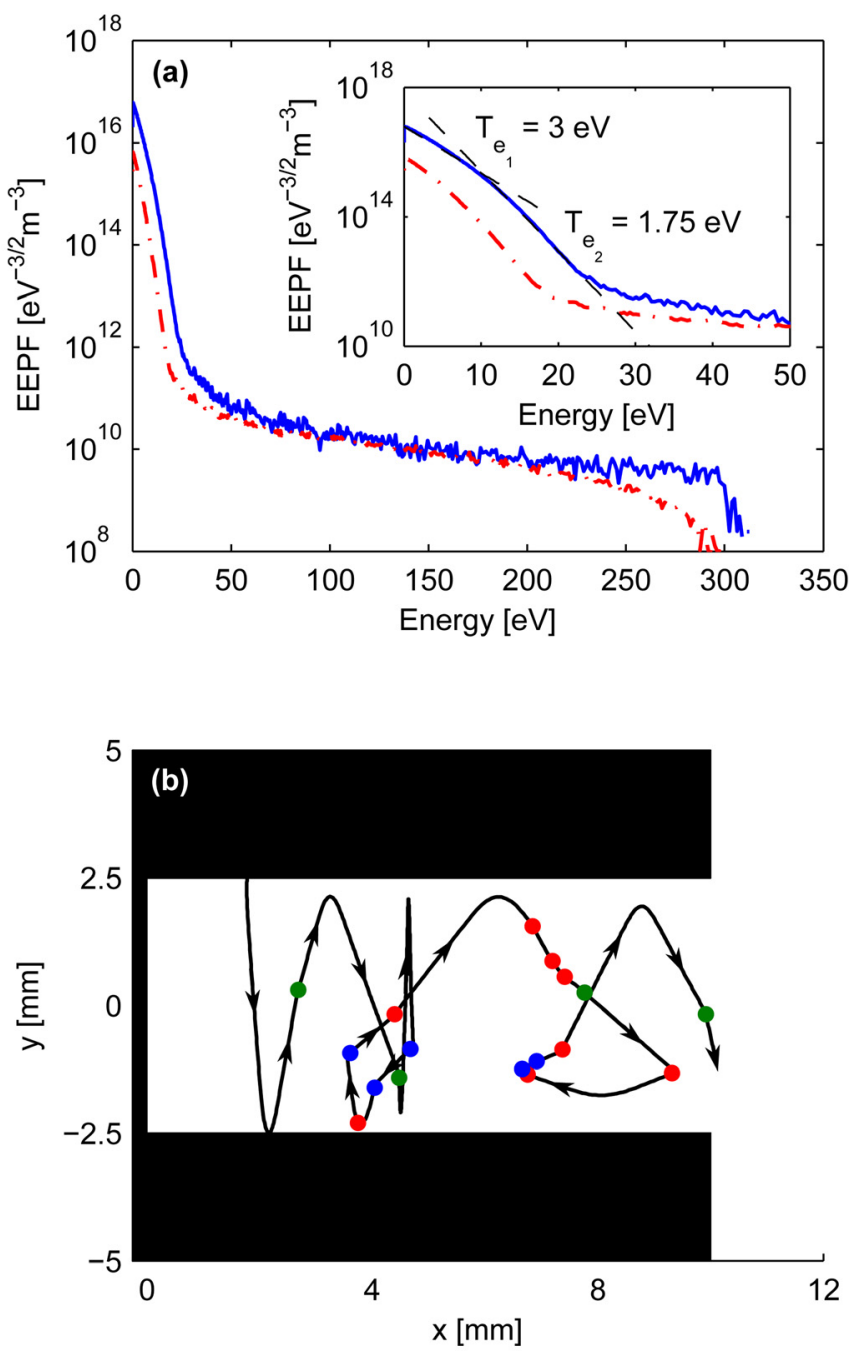

FIG. 13. (Color online) (a) Time-averaged electron energy probability function (EEPF) for case 4 in Fig. 4. The solid line shows the EEPF at $x=5 \mathrm{~mm}$ and $y=0 \mathrm{~mm}$ (closed circle in Fig. 6), while the dashed lines show the EEPF at $x=5 \mathrm{~mm}$ and $y=2.25 \mathrm{~mm}$ (open circle in Fig. 6). The inset figure shows a magnified view of the EEPF over a range of $0-50 \mathrm{eV}$. (b) Example trajectory of a secondary electron within the HC discharge collected over $7.5 \mathrm{~ns}$. The arrows indicate the electron's direction of motion, while the closed circles indicate the location of an electron-neutral collision, with elastic (red online), excitation (green online), and ionization (blue online). The black regions show the geometry and location of the powered electrode.

dominated by capacitive reactance. By finding the current density and using the area for electrodes with a radius of $70 \mathrm{~mm}$ (as used in Ref. 10), we obtain a total RMS discharge current of about $4.9 \mathrm{~A}$, which is within the range of values $(1-9 A)$ used in Ref. 10. Due to the presence of the bias capacitor, the average current is $0 \mathrm{~A}$ (i.e., there is no $\mathrm{dc}$ component).

Since the simulation circuit shown in Fig. 1(b) is a series circuit, the current at both the grounded and powered electrodes must be equal to the current at any other point in the external circuit. The thin solid curve in Fig. 14(a) shows the current obtained from the external circuit model of the simulation, while the closed circles show the total current at the powered electrode, found by summing the total conduction and displacement currents. As is seen, both currents match very closely, which serves as a good consistency check of
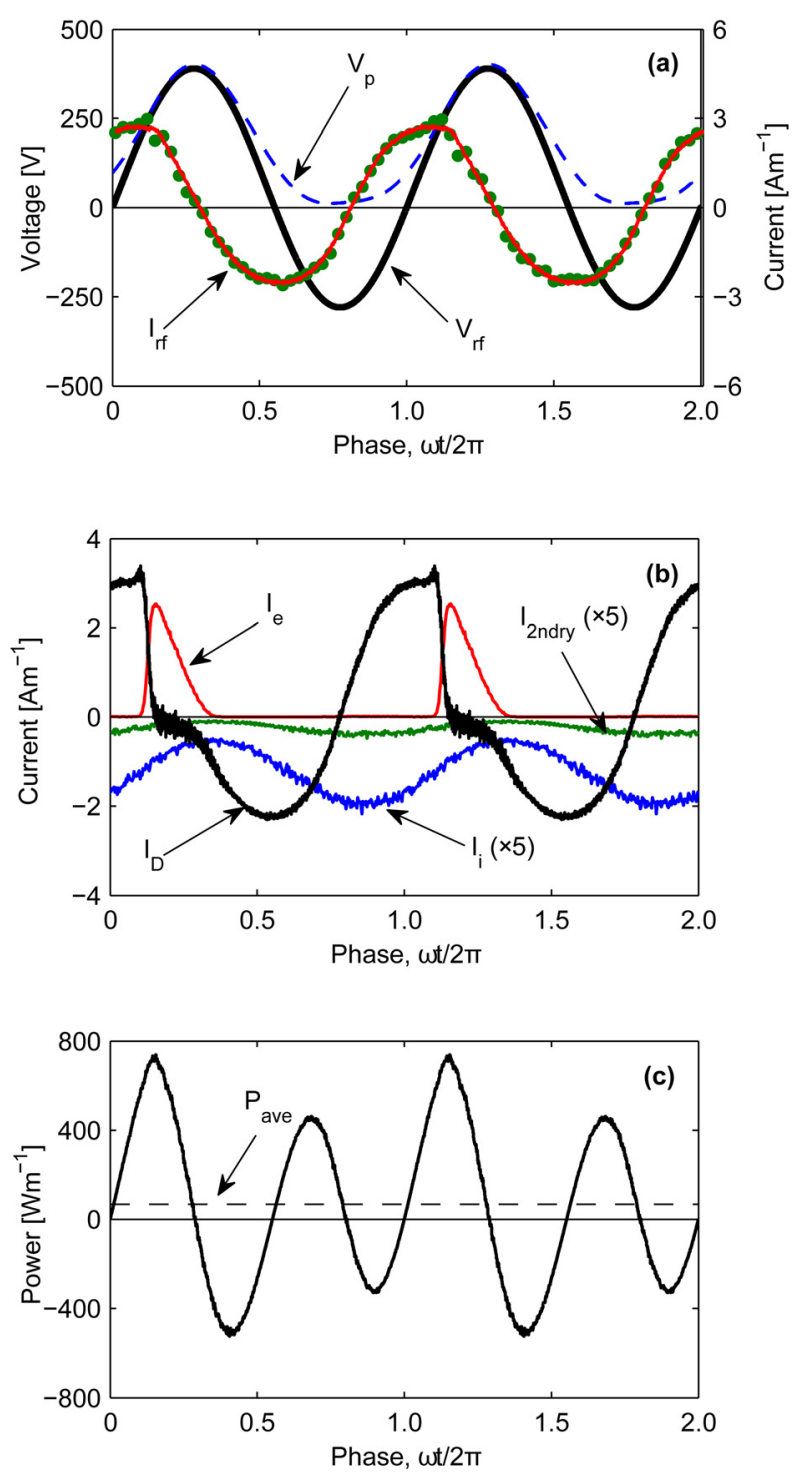

FIG. 14. (Color online) (a) Powered electrode voltage ( $V_{r f}$, thick solid line), plasma potential $\left(V_{p}\right.$, at $x=5 \mathrm{~mm}$ and $y=0 \mathrm{~mm}$; dashed line), and total discharge current ( $I$, thin solid line and closed circles). The current given by the thin solid line is found from the external circuit model, while the current given by the closed circles is found from the sum of the current components at the powered electrode (see part (b)). (b) Current composition at the powered electrode, showing the displacement current $\left(I_{D}\right)$, electron current $\left(I_{e}\right)$, ion current $\left(I_{i}\right)$, and the secondary electron current $\left(I_{2 n d r y}\right)$. The ion and secondary electron currents have been scaled by a factor of 5 in order to show the rf modulation more clearly. (c) Instantaneous power absorption within the discharge as a function of rf phase. The horizontal dashed line shows the time-averaged power absorption. To aid clarity, the results in (a)-(c) have been plotted over $2 \mathrm{rf}$ periods.

the simulation. In order to find the total displacement current at the powered electrode, $I_{D}(t)$, the Ampere-Maxwell equa$\operatorname{tion}^{36}$ (in integral form) is used:

$$
\oint \mathbf{B} \cdot d \mathbf{l}=\mu_{0} I_{c}+\mu_{0} \epsilon_{0} \int \frac{\partial \mathbf{E}}{\partial t} \cdot d \mathbf{A}=\mu_{0} I_{c}+\mu_{0} I_{D},
$$

where $\mathbf{B}$ is the magnetic field (produced by a current carrying wire; this is not modelled in the simulation, nor is it needed), $d \mathbf{l}$ is an element of length along the integration path (Amperian loop) in Fig. 1(b), $\mu_{0}$ is the permeability of free space, $I_{c}$ is 
the conduction current, $\mathbf{E}$ is the electric field, $A$ is the area of the surface bounded by the loop in Fig. 1(b), and $I_{D}$ is the displacement current. Shown in Fig. 1(b) is an Amperian loop bounding two surfaces. For surface $1, I_{c}=I, \mathbf{E}=0$, and thus $\oint \mathbf{B} \cdot d \mathbf{l}=\mu_{0} I$, while for surface 2 surrounding the powered electrode, $\quad \oint \mathbf{B} \cdot d \mathbf{l}=\mu_{0} I_{c}+\mu_{0} I_{D}$. Thus, $\quad I=I_{c}+I_{D}=I_{c}$ $+\epsilon_{0} \int \partial \mathbf{E} / \partial t \cdot d \mathbf{A}$. By using the right-hand rule, the thumb points in the direction of the outward normal $d \mathbf{A}$ along the surface surrounding the powered electrode. ${ }^{36}$ This sign consistency is needed to correctly calculate the displacement current at the powered electrode, which is found by discretising $\partial \mathbf{E} / \partial t$ and summing the contribution from each portion of the electrode.

Figure 14(b) shows the components of the total current at the powered electrode over $2 \mathrm{rf}$ periods. Note that due to the positive current direction chosen in Fig. 1(b), ions (and also secondary electrons emitted from the electrode) that strike the powered (left) electrode constitute a negative current, while electrons striking the electrode constitute a positive current. The displacement current, $I_{D}$, is seen to be approximately sinusoidal, except near $\omega t / 2 \pi=0.15$, where it drops sharply. This sharp drop is correlated with a large electron current, $I_{e}$, occurring in pulses synchronised with the $\mathrm{rf}$, as the sheath collapses. The ion current, $I_{i}$, and ion produced secondary current, $I_{2 n d r y}$, are both shown magnified by a factor of 5 . These currents, while present throughout the rf cycle, are seen to be significantly modulated by the rf. The time-averaged sheath width within the slit is about $0.86 \mathrm{~mm}$ and ions within the sheath have a spread of energies between 0 and $80 \mathrm{eV}$. Ions with an average energy greater than $30 \mathrm{eV}$ have a transit time within the sheath of less than an rf period, and thus a modulation of the ion current is expected. The total secondary electron current is found to be $20 \%$ that of the total ion current, consistent with the secondary electron emission coefficient of 0.2 used in the simulation. The results presented in Figs. 14(a) and 14(b) are similar to those observed in standard CCP discharges. ${ }^{29,34}$

The instantaneous power deposited into the discharge can be found from $P(t)=V(t) I(t)$ and is plotted in Fig. 14(c). Since both the electrode voltage and total current vary almost sinusoidally, the instantaneous power oscillates at twice the applied rf frequency. The black dashed line in Fig. 14(c) shows the time-averaged power into the discharge, which is about $67.5 \mathrm{Wm}^{-1}$. By finding the power per unit area and using the area of an electrode with a radius of $70 \mathrm{~mm},{ }^{10}$ we obtain a total discharge power of about $200 \mathrm{~W}$, which is again within the range of values (about $50-400 \mathrm{~W}$ ) used in Refs. 10 and 30.

\section{DISCUSSION}

The results presented above in Sec. IV clearly, and consistently, demonstrate the importance of secondary electron emission in sustaining the HC discharge. In particular, secondary electron emission due to ion bombardment is required, and electron produced secondaries were found to have little effect on the discharge. This can be understood by observing Fig. 14, where the electrons are lost in pulses at the powered electrode when the sheath collapses. Thus, elec- tron produced secondaries would also be generated in pulses at this rf phase. However, from Fig. 7(b), at this point in the rf cycle, we see that the accelerating voltage, $\Delta V$, is close to zero. Therefore, these secondaries are not accelerated to very high energies. With ion produced secondaries, however, even though the ion current (and hence secondary current) is modulated, there is always a current present throughout the rf cycle. During the negative portion of the rf cycle then, secondaries can be accelerated across the large voltage difference, $\Delta V$, to very high energies. These electrons, as seen in Fig. 13(b), can then oscillate between opposite walls of the slit, suffering multiple inelastic collisions before being lost from the system.

In standard CCP discharges, it is well known that secondary electrons can have an effect even in the absence of a $\mathrm{HC}$ type geometry. However, the effect of secondaries is usually only important at lower frequencies or much higher pressures. $^{29}$ At the usual operating frequency of $13.56 \mathrm{MHz}$ and pressures of about $250 \mathrm{mTorr}$, the discharge is essentially sustained by stochastic sheath heating. In the simulation context, the plane electrode case (case 1) is representative of a standard CCP discharge, and while secondary electron emission is present, as was observed in the ionization and power absorption characteristics the discharge primarily displays rf sheath heating behaviour. For the same operating parameters, however, the HC discharge (case 4) showed very different behaviour, with features characteristic of secondary electron heated discharges. Furthermore, with secondaries turned off, the plasma was seen to leave the slit and the density decreased. This suggests that sheath heating is not strong enough to sustain the $\mathrm{HC}$ discharge and can be understood from the results in Fig. 10. Here, the power absorption in region 3 was equal in magnitude to the negative power absorption in region 2 . Thus, the net power absorption at the sheath edge is 0 , and the discharge is sustained entirely by secondary heated electrons.

Although comparison of the simulation results with the experimental measurements in Ref. 10 showed favourable agreement, due to the larger densities at higher voltages (and consequently the larger grid point numbers, particles, and smaller time step needed), it was not possible to perform simulations over a larger parameter range. Indeed, with the time step and grid point numbers used, numerical heating was observed at large voltages $(>500 \mathrm{~V})$ and higher pressures ( $\sim 500$ mTorr). This is because the plasma density gets too high, so that in order to resolve the Debye length and plasma period, much smaller time steps and increased grid point numbers are needed, which consequently results in more particles being needed, and much longer run times. While reasonable simulation results can sometimes still be achieved even in the presence of some numerical heating (typically manifesting as increased power absorption within the lower energy electron population ${ }^{37}$ ), since the density rises so sharply in Fig. 5, numerical heating was observed to become too large at the higher voltages for useful results to be obtained. Future work will aim to adopt a parallel or graphics processing unit (GPU) computing approach to reduce run times, as well as improving the simulation by incorporating an energy dependent secondary electron model and investigating a larger parameter space. 
Finally, although we have presented strong evidence above to show that $\mathrm{rf} \mathrm{HC}$ discharges are sustained by secondary electron heating, we cannot conclude that this is true for all parameters and geometries. The presence of positive power absorption at the sheath edge in Fig. 10 suggests that perhaps at higher frequencies or different geometric conditions, it might be possible to enhance the sheath heating component and reduce the secondary electron heating component. There is some recent experimental evidence ${ }^{38}$ suggesting that this change in the heating mechanism might be possible, where indirect signs of sheath heating were observed by analysing the harmonic content of the current waveform in an rf micro-HC. Since the excitation crosssection for argon is similar to the ionization cross-section, more concrete experimental evidence might be obtained by observing the light emission from a $\mathrm{HC}$ discharge in relation to the rf phase, similarly to that in Fig. 7, where secondary heated and sheath heated discharges produced different behaviour.

\section{CONCLUSIONS}

In summary, a two-dimensional PIC-MCC code has been developed and used to simulate a capacitively coupled rf discharge. The presence of a multi-slit powered electrode is observed to enhance the plasma density and results from a hollow cathode discharge that is sustained through secondary electron emission due to ion bombardment. The energy of these secondary electrons is seen to be strongly modulated by the applied rf potential, and they are observed to oscillate back and forth between opposite walls within the slit. By investigating the ionization and power absorption profiles, $\mathrm{rf}$ sheath heating is found to be too weak to both initiate and sustain the discharge; secondary electron acceleration across the sheath is the primary power deposition mechanism.

\section{ACKNOWLEDGMENTS}

The authors gratefully acknowledge financial support from the Lam Research Corporation.

${ }^{1}$ L. Bárdos, Surf. Coat. Technol. 86-87, 648 (1996).

${ }^{2}$ L. Bárdos, H. Baránková, and S. Berg, Surf. Coat. Technol. 97, 723 (1997).

${ }^{3}$ J. V. Sullivan and A. Walsh, Spectrochim. Acta 21, 721 (1965).
${ }^{4}$ D. M. Goebel and I. Katz, Fundamentals of Electric Propulsion: Ion and Hall Thrusters (John Wiley \& Sons, New Jersey, 2008).

${ }^{5}$ M. C. Storrie-Lombardi, W. F. Hug, G. D. McDonald, A. I. Tsapin, and K. H. Nealson, Rev. Sci. Instrum. 72, 4452 (2001).

${ }^{6}$ R. M. Sankaran and K. P. Giapis, J. Appl. Phys. 92, 2406 (2002).

${ }^{7}$ L. Bárdos, S. Berg, and H. O. Blom, Appl. Phys. Lett. 55, 1615 (1989).

${ }^{8}$ M. Mildner, D. Korzec, and J. Engemann, Surf. Coat. Technol. 112, 366 (1999).

${ }^{9}$ H. Baránková and L. Bárdos, Surf. Coat. Technol. 146-147, 486 (2001).

${ }^{10}$ H. S. Lee, Y. S. Lee, S. H. Seo, and H. Y. Chang, Thin Solid Films 519, 6955 (2011).

${ }^{11}$ H. S. Lee, Y. S. Lee, S. H. Seo, and H. Y. Chang, Appl. Phys. Lett. 97, 081503 (2010).

${ }^{12}$ V. I. Kolobov and L. D. Tsendin, Plasma Sources Sci. Technol. 4, 551 (1995).

${ }^{13}$ R. R. Arslanbekov, A. A. Kudryavtsev, and R. C. Tobin, Plasma Sources Sci. Technol. 7, 310 (1998).

${ }^{14}$ P. Gessini, S. B. Gabriel, and D. G. Fearn, in 27th International Electric Propulsion Conference, Pasadena California, 15-19 October, IEPC-01233 (Electric Rocket Propulsion Society, Ohio, 2001).

${ }^{15}$ R. A. Arakoni, J. J. Ewing, and M. J. Kushner, J. Phys. D: Appl. Phys. 41, 105208 (Electric Rocket Propulsion Society, Ohio, 2008).

${ }^{16}$ P. S. Kothnur and L. L. Raja, J. Appl. Phys. 97, 043305 (2005).

${ }^{17}$ J. P. Boeuf, L. C. Pitchford, and K. H. Schoenbach, Appl. Phys. Lett. 86, 071501 (2005).

${ }^{18}$ N. Baguer, A. Bogaerts, Z. Donko, R. Gijbels, and N. Sadeghi, J. Appl. Phys. 97, 123305 (2005).

${ }^{19}$ C.K. Birdsall and A. B. Langdon, Plasma Physics Via Computer Simulation (McGraw-Hill, New York, 1985).

${ }^{20}$ G. J. Kim, F. Iza, and J. K. Lee, J. Phys. D: Appl. Phys. 39, 4386 (2006).

${ }^{21}$ Y. Miyagawa, H. Nakadate, M. Tanaka, M. Ikeyama, and S. Miyagawa, Surf. Coat. Technol. 186, 2 (2004).

${ }^{22}$ G. J. Kim and J. K. Lee, IEEE Trans. Plasma Sci. 36, 1238 (2008).

${ }^{23}$ V. Vahedi and M. Surendra, Comp. Phys. Commun. 87, 179 (1995).

${ }^{24}$ M. Hayashi, J. Phys. D 15, 1411 (1982).

${ }^{25}$ J. Ferch, B. Granitza, C. Masche, and W. Raith, J. Phys. B 18, 967 (1985).

${ }^{26}$ F. J. de Heer, R. H. J. Jansen, and W. van der Kaay, J. Phys. B 12, 979 (1979).

${ }^{27}$ E. Krishnakumar and S. K. Srivastava, J. Phys. B 21, 1055 (1988).

${ }^{28}$ A. V. Phelps, J. Appl. Phys. 76, 747 (1994).

${ }^{29}$ M. A. Lieberman and A. J. Lichtenberg, Principles of Plasma Discharges and Materials Processing (John Wiley \& Sons, New Jersey, 2005).

${ }^{30} \mathrm{H}$. Y. Chang, private communication (2011).

${ }^{31}$ V. Vahedi and G. DiPeso, J. Comput. Phys. 131, 149 (1997).

${ }^{32}$ E. Kawamura, C. K. Birdsall, and V. Vahedi, Plasma Sources Sci. Technol. 9, 413 (2000).

${ }^{33}$ L. Bárdos and N. Q. Vu, Czech. J. Phys. B 39, 735 (1989).

${ }^{34}$ D. Vender and R.W. Boswell, IEEE Trans. Plasma Sci. 18, 725 (1990).

${ }^{35}$ M. Surendra and D. B. Graves, IEEE Trans. Plasma Sci. 19, 144 (1991).

${ }^{36}$ D.J. Griffiths, Introduction to Electrodynamics, 3rd ed. (Prentice-Hall, New Jersey, 1999).

${ }^{37}$ V. Vahedi, G. DiPeso, C.K. Birdsall, M. A. Lieberman, and T. D. Rognlien, Plasma Sources Sci. Technol. 2, 261 (1993)

${ }^{38}$ C. M. O. Mahony, T. Gans, W. G. Graham, P. D. Maguire, and Z. Lj. Petrović, Appl. Phys. Lett. 93, 011501 (2008). 